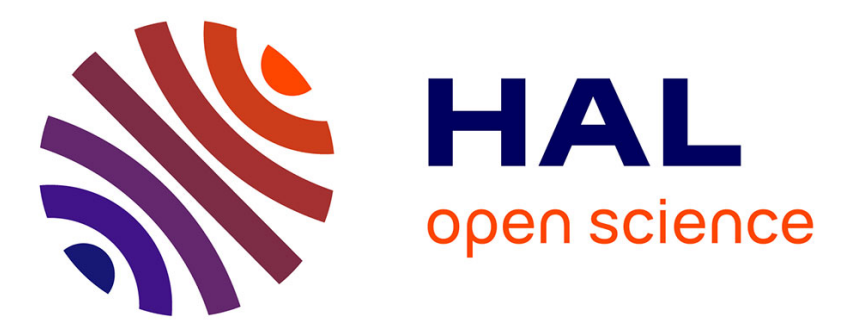

\title{
Demonstration of a new technology which allows direct sensor integration on flexible substrates
}

\author{
A. Petropoulos, D. Goustouridis, T. Speliotes, G. Kaltsas
}

\section{To cite this version:}

A. Petropoulos, D. Goustouridis, T. Speliotes, G. Kaltsas. Demonstration of a new technology which allows direct sensor integration on flexible substrates. European Physical Journal: Applied Physics, 2009, 46 (1), pp.1-4. 10.1051/epjap/2009020 . hal-00480150

\section{HAL Id: hal-00480150 \\ https://hal.science/hal-00480150}

Submitted on 3 May 2010

HAL is a multi-disciplinary open access archive for the deposit and dissemination of scientific research documents, whether they are published or not. The documents may come from teaching and research institutions in France or abroad, or from public or private research centers.
L'archive ouverte pluridisciplinaire HAL, est destinée au dépôt et à la diffusion de documents scientifiques de niveau recherche, publiés ou non, émanant des établissements d'enseignement et de recherche français ou étrangers, des laboratoires publics ou privés. 


\author{
Demonstration of a New Technology Which Allows Direct Sensor \\ INTEGRATION ON FLEXIBLE SUBSTRATES \\ Anastasios Petropoulos ${ }^{1}$, Dimitrios Goustouridis ${ }^{1}$, Thanassis Speliotes ${ }^{2}$, Grigoris Kaltsas ${ }^{*}, 3,1$ \\ ${ }^{1}$ Institute of Microelectronics NCSR Demokritos, Athens, Greece \\ ${ }^{2}$ Institute of Materials Science NCSR Demokritos, Athens, Greece \\ ${ }^{3}$ Department of Electronics, TEI of Athens, Aegaleo, Greece \\ *Corresponding author e-mail: G.Kaltsas@ee.teiath.gr
}

\begin{abstract}
In this work we present a fabrication method for developing thermal sensors on flexible organic substrates. The constructed devices consist of Pt thermistors which are directly integrated to the copper tracks of a flexible copper-clad laminate. They reside on top of a $12 \mu \mathrm{m}$ thick SU-8 planarization layer, while a sacrificial layer utilized by the negative photoresist ma$N$ was used in order to define the thermistor pattern. The thermistors can act as both heating and temperature sensing elements, while due to small thickness and the low thermal conductivity of the Kapton substrate a very effective thermal isolation is achieved. The minimum radius of curvature of the fabricated devices was found to be $5 \mathrm{~mm}$. As the device is in direct communication to the macrowolrd, the need for wire bonding is eliminated, while the final surface of the produced sensor is relatively planar. The overall process is simple and cost-effective with minimal requirements in fabrication time. The potential application field of the presented devices is considered quite extensive as they can be directly expanded into flexible sensors able to measure quantities such as fluid flow rate, displacement or vacuum.
\end{abstract}

\section{Introduction}

The commercial availability of metalized flexible organic films introduced an increasing effort in using these substrates for the fabrication of various flexible devices [1]. Typically, the desired patterns are formed by chemical etching of the metal on selected areas. However, the resulting surface topology induces major restrictions on further process flow steps, thus limiting the fabrication of additional device structures. The technology proposed here provides the ability for microstructure formation on top of macro-structured flexible substrates. Thus a micro-device can be integrated using standard microsystem techniques on top of a flexible platform that implements the corresponding control and read out electronics.

\section{Fabrication method \\ 2.1 Overview}

Recently, a novel fabrication method which concerns the formation of thermal sensors on printed circuit boards was presented [2]. The method which is described in this paper extends the specific technology to the formation of micro-devices in flexible substrates. The main problem regarding the integration of microstructures using lithography on commercial copper clad composites is the remaining topography on the substrate surface after the definition of the copper tracks, which is of the order of $10 \mu \mathrm{m}$. The proposed method involves the formation of an SU-8 planarization layer on the substrate surface which aims to provide a smooth path for a subsequently deposited thin second metal film. The micro-device pattern is then defined by performing lithography on a sacrificial layer composed by the negative tone phototresist ma-N. The final device is produced after the second metal film deposition and the subsequent lift-off process. A layout of the process flow

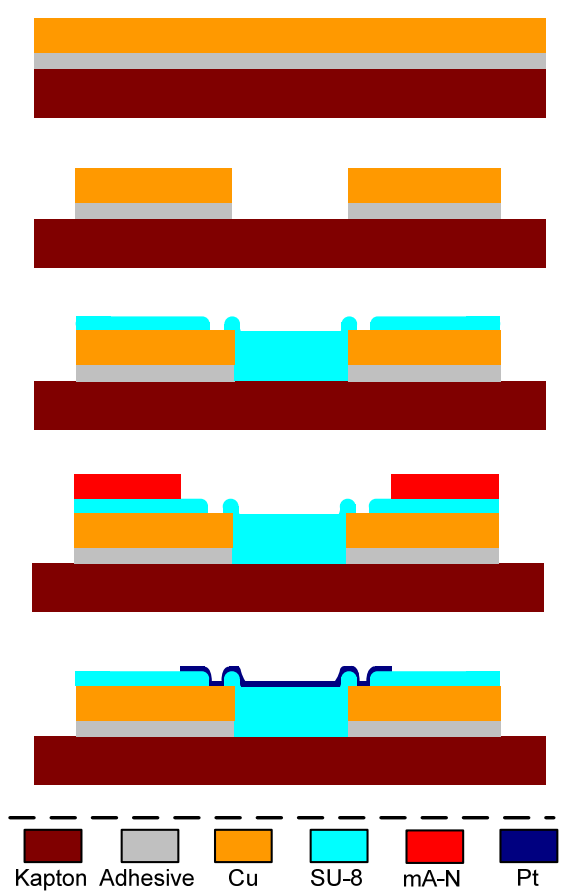

Fig 1. The fabrication sequence:

a) The copper clad laminated Kapton composite

b) Chemical etching of copper

c) Formation of the SU-8 planarization layer

d) Formation of the ma-N sacrificial layer

e) Pt sputtering and lift-off. The final form of the device 

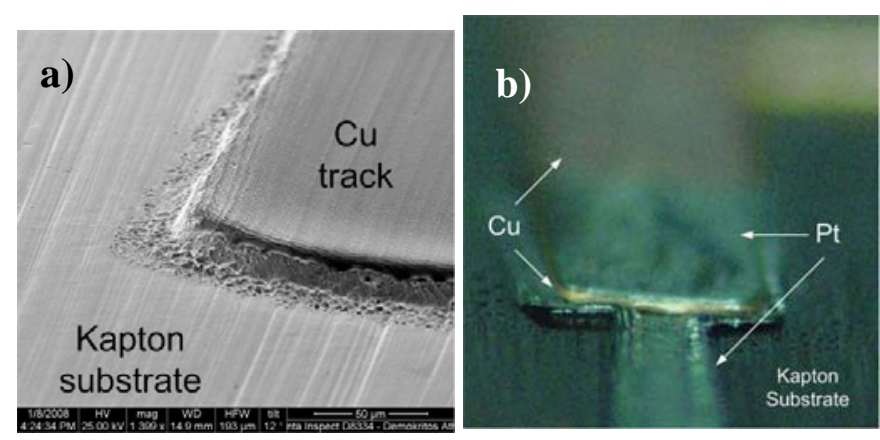

Fig 2. a) SEM image of the edge of the copper structure. b) Direct deposition of $\mathrm{Pt}$ on the pre-patterned organic substrate. The discontinuity of the Pt film at the edge of the copper track is clearly indicated.

is shown in Fig. 1. The fabricated sensors consist of thin Pt films which electrically connect distinct copper tracks directly defined on the organic substrate. The fabricated thermistors can act as both heating elements by utilizing the Joule effect, or as temperature sensing elements.

Regarding the involved lithography steps, high quality laser-printed transparencies were used in order to define the desired structures instead of the standard quartz photomasks. The minimum reproducible feature size obtained was $100 \mu \mathrm{m}$. The use of transparencies allows a significant reduction of the involved cost, while the required time to implement a new design is minimal. These characteristics are highly beneficial since the proposed fabrication technology aims to provide a platform for the production of varying types of thermal sensors.

\subsection{Copper pattern formation}

The initial device substrate is a Pyralux ${ }^{\circledR}$ copper-clad laminated composite, which is constructed of DuPont Kapton ${ }^{\circledR}$ polyimide film with a $12.5 \mu \mathrm{m}$ thick copper foil on one side. In order to perform the necessary lithography steps, the flexible substrate is mounted onto a carrier wafer. The positive photoresist AZ was used for the pattern definition of the copper structures, while subsequent chemical etching removed the copper from the unprotected areas.

\subsection{The Planarization layer}

Direct metal deposition in order to establish electrical connections between distinct copper tracks is not applicable, due to the size and nature of the sidewalls of the copper structures. Furthermore, existing residuals of the adhesive sheet which bonds the copper foil to the polymide substrate introduce further anomalies on the surface as shown in Fig 2a. Fig $2 b$ shows the outcome of direct $\mathrm{Pt}$ deposition on the pre-defined flexible substrate, whereby the electrical continuity of the $\mathrm{Pt}$ film is destroyed at the edge of the copper track.

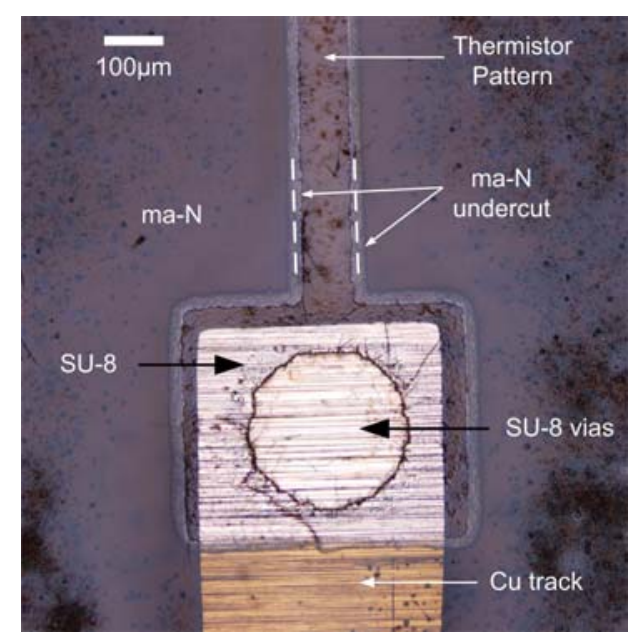

Fig 3. An optical microscope image of the fabricated structures prior to the Pt deposition

In order to provide a smooth path for the subsequently deposited metal lines, a planarization layer utilized by the thick negative photoresist SU-8 is spin coated on the patterned substrate. The planarization layer is extended so as to cover the edges of the copper tracks. The contact of the subsequently deposited thin Pt film to the copper tracks is performed through vias that are formed in the SU-8 layer on top of the copper tracks. The selection of the specific photoresist as the planarization layer material was based on the fact that it exhibits excellent adhesion to both the copper and the polymide surface, while it presents high resistivity to etchants. The exposure dose and the corresponding layer thickness are also significant parameters regarding the formation of a functional planarization layer. The proper tuning of these parameters requires the consideration of factors such as the difference in the reflectivity between the copper and the polymide substrate as well as the SU8 layer non-uniformity in terms of thickness. The exposure dose window is quite narrow, as a potential overexposure of the photoresist leads to poor definition of the vias. Furthermore, underexposed SU-8 cannot survive the subsequent lift-off stage due to the uncompleted cross-linking process. The layer thickness used in the formation of the devices presented here was $12 \mu \mathrm{m}$, with a corresponding exposure energy of 240 $\mathrm{mJ} / \mathrm{cm}^{2}$.

\subsection{Sacrificial layer and lift off}

The perquisite regarding the photoresist used as sacrificial layer is to span a thickness range which is compatible to the rest of the process. Photoresists used for lift-off purposes are typically of the order of $1 \mu \mathrm{m}$ thick. Since the structures on the device surface impose thickness variations exceeding $10 \mu \mathrm{m}$, the use of such photoresists does not provide a sacrificial layer of adequate homogeneity. The photoresist used in this case, was the negative tone ma-N 400 which exhibits a 


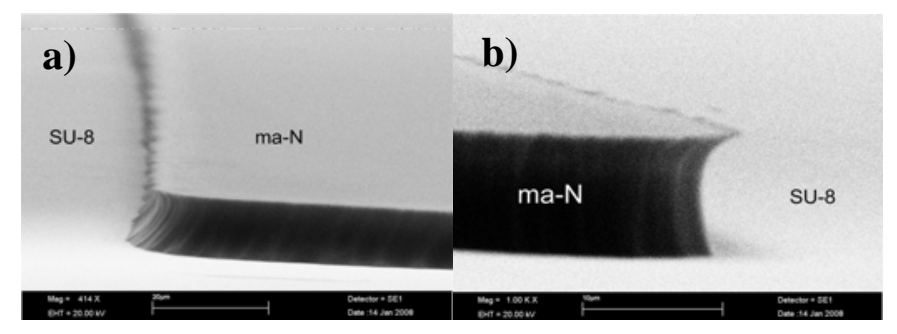

Fig 4. SEM images of ma-N structures of $7.5 \mu \mathrm{m}$ thickness. The effect of development time in the formation of the undercut is shown. a) Development time $140 \mathrm{sec}, \mathrm{b})$ Development time $170 \mathrm{sec}$

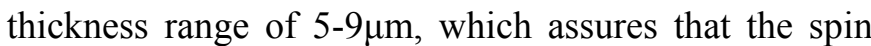
coating over the existing structures is of adequate quality (Fig. 3). Furthermore, the fact that the photoresist is of negative tone allows the formation of negative sidewalls, so that the thermistor structures can be accurately defined. The factors affecting the wall gradient are the exposure dose and primarily the development time. For a specified exposure dose, a larger developing time leads to the removal of the less exposed part of the photoresist near the bottom of the structures, leading to the formation of an undercut. This can be seen in the SEM images of fig 4, where two structures of identical thickness are shown. The structure of fig $4 \mathrm{~b}$ however has remained in the developer solution longer; this way the desired undercut is achieved. Actually a rather accurate estimation regarding the size of the undercut can be maintained during the developing process. In the bright field optical microscopy image shown in Fig. 3, the undercut is depicted as the thin pale line at the border of the ma-N structure. The line width is a function of the developing time. The latter is a significant point regarding the yield provided in the subsequent lift- off stage, as it allows the correction of effects that might be induced on the structures by certain critical process parameters affecting the thickness uniformity of the sacrificial layer. Such parameters are the exposure dose which may not be spatially uniform or the surface of the flexible substrate which may not be totally flat throughout the spinning process.

\subsection{Second metal deposition}

Following the formation of the sacrificial layer, a bilayer of Ti/Pt of $30 / 300 \mathrm{~nm}$ thickness is sputtered on the surface. Finally the lift-off stage is taking place, where the sacrificial layer and the metal residing on it are being removed by inserting the structure into acetone. There are two factors associated to the success of the lift off stage. The first one concerns the SU-8 layer, which has to be fully crosslinked in order to avoid the compromise of its adhesion to the substrate, while the second one is the need for negative sidewalls on the patterned ma-N structures.

\section{The final device}

\subsection{Device Characteristics}

In order to demonstrate the validity of the proposed technology an array of temperature sensors were fabricated and evaluated on top of a flexible organic substrate. A photograph of the fabricated device is shown in Fig 5. The sensors' lateral dimensions are $1.5 \times 0.1 \mathrm{~mm}^{2}$. The fabricated thermistors can act both as heating or temperature sensing elements with a corresponding TCR of $0.0025 /{ }^{\circ} \mathrm{C}$. As it can be seen from fig. 5, the device can bend with regard to two axes of curvature. The sensing elements of the device are directly integrated to the circuit electronics; therefore there is no need for wire bonding which makes the device suitable for use in harsh environments. The final surface is relatively planar which allows for further lithography steps to be performed, but it can also provide minimally invasive measurements when the device is used as a flow sensor. The overall fabrication procedure is very cost-effective, with reduced equipment requirements as it can be carried out outside the clean room. Finally, the simplicity of the process implies that the device fabrication time is kept to a very low level.

The implementation of an alternative version of the presented technology [2] on rigid organic substrates has already produced a family of PCB-based sensors with extremely satisfying operational characteristics. All sensors employ the monitoring of heat transfer to the thermistors in order to measure quantities such as the gas flow rate, the liquid flow rate, vacuum, position and microchannel flow [3,4]. Flexible thermal sensors measuring the aforementioned quantities can be directly fabricated, with the potential of an expanded application field, for instance the measuring the gas and liquid flow on curved surfaces.

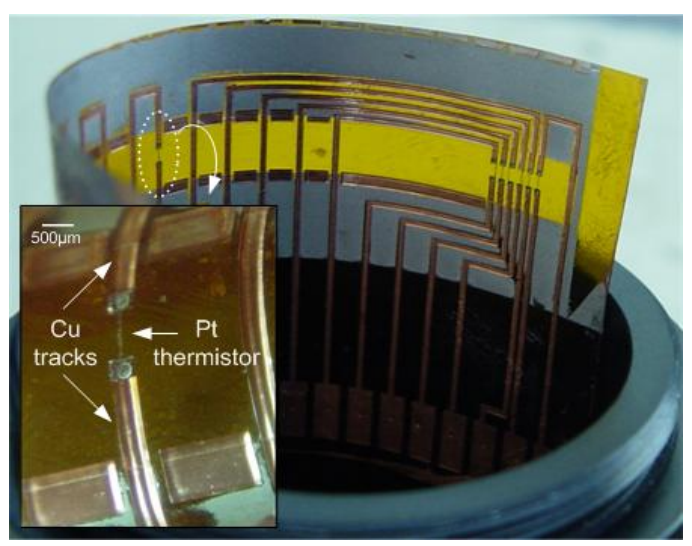

Fig 5. A photograph of the final device. 


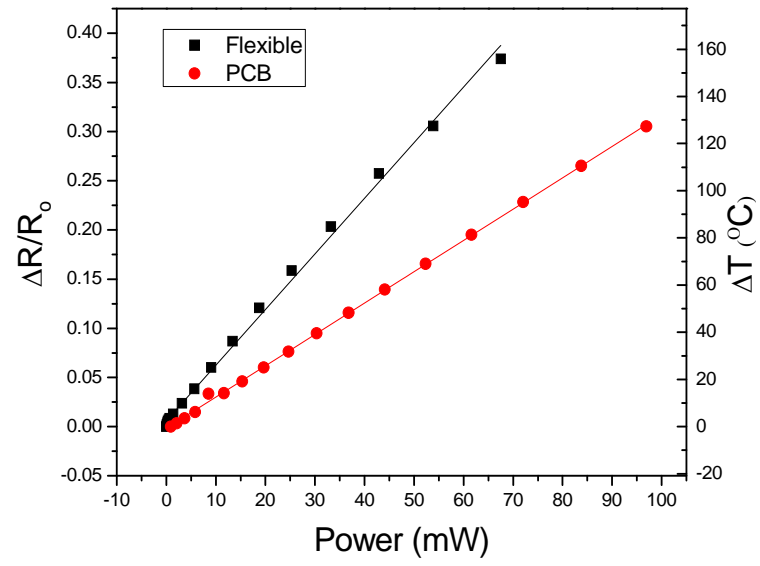

Fig 6. The temperature increase as a function of power regarding a thermistor fabricated on a flexible substrate and a similar one of the same dimensions fabricated on a rigid PCB

\subsection{Mechanical operating limits}

The mechanical strength of the devices is mainly defined by the SU-8 layer. The mechanical properties of SU-8 are dependent on process parameters such as the baking temperature, baking time and the UV dosage [5]. Typical values are a Young's modulus of 4.02 GPa [6] and a tensile strength of $40-60 \mathrm{MPa}$ [5]. Since the corresponding tensile strength for copper is $220 \mathrm{MPa}$ and 234Mpa for Kapton and alongside the fact that the thickness of the SU-8 layer is $12 \mu \mathrm{m}$ which can be considered a relatively large value, the planarization layer constitutes the less flexible part of the device. The minimum radius of curvature was experimentally established as $5 \mathrm{~mm}$. There is ongoing effort regarding the potential to minimize the layer thickness and subsequently the minimum radius of curvature. It has to be noted that, although the SU-8 layer thickness is $12 \mu \mathrm{m}$, on top of the copper structures it reduces down to $5 \mu \mathrm{m}$ which makes it more flexible and therefore prevents potential detachment.

\subsection{Thermal properties}

Thermal sensors in general aim to measure a large number of quantities. Typically, they contain a heating element which gives rise to an increased temperature field and sensing elements that monitor the change in the temperature field as induced by the quantity that is measured. Heat conduction from the heating element to the substrate has a negative impact on the device resolution, sensitivity and measurement range. MEMS thermal sensors are typically fabricated on Si substrate, which exhibits a high thermal conductivity value. In order to address this issue, a number of isolation schemes have been presented in the literature such as the formation of free standing structures, vacuum cavities, the use of porous Si [7] etc. However these are quite often demanding process steps which might also introduce limitation to further lithography steps.

In this case however the materials that comprise the fabricated devices exhibit very low thermal conductivity values, which are $0.2 \mathrm{~W} / \mathrm{m} \cdot \mathrm{K}$ for SU-8 and $0.12 \mathrm{~W} / \mathrm{m} \cdot \mathrm{K}$ for Kapton ${ }^{\circledR}$. Furthermore the overall thickness of the device is less than $100 \mu \mathrm{m}$ signifying a very effective thermal isolation of the heating element. In order to quantify the specific effect, the heating element temperature increase caused by the application of a specific amount of power was calculated and compared to corresponding temperature increase induced to a similar Pt thermistor fabricated on a rigid PCB. The latter is composed by FR4 which exhibits a thermal conductivity value of $0.2 \mathrm{~W} / \mathrm{m} \cdot \mathrm{K}$, but is $2 \mathrm{~mm}$ thick. It was found that the temperature increase of the thermistor fabricated on the flexible substrate was $77 \%$ higher than that of a corresponding thermistor fabricated on a regular $\mathrm{PCB}$, referring to the same power values (Fig 6). This fact implies that the effective thermal isolation of the fabricated thermistors is very high, with potential gain in the operational characteristics of the devices.

\section{Conclusions}

A new technology which allows the fabrication of micro-devices directly on flexible substrates was demonstrated. The proposed technique combines microsystem technology and standard PCB methods. The demonstration of the proposed technology was made through the fabrication of specific devices which are directly connected to macroscale copper structures of commercial flexible copper-clad laminated composites. The constructed Pt thermistors can act as heating or temperature sensing elements, fully integrated to the substrate electronics. A very high degree of thermal isolation of the thermistors is achieved, which makes them suitable for a variety of thermal sensing applications.

\section{Acknowledgements}

This work is co-funded by E.U.- European Social Fund (75\%) and the Greek Ministry of Development-GSRT (25\%) under the framework of the research program PENED.

\section{References}

[1] D.J. Lictenwalner et. Al, Sens. Actuators A, 135, (2007)

[2] G. Kaltsas et.al, J. Phys. Conf. Series, 92, 012046, (2007)

[3] A. Petropoulos et. al., Phys. Status Solidi a, submitted

[4] A. Petropoulos et. al., Microelectron. J., doi:10.1016/j.mejo.2008.04.015, (2008)

[5] R. Feng and R.J. Farris, J. Micromech. Microeng., 13, (2003)

[6] H. Lorenz et. al, J. Micromech. Microeng., 7, (1997)

[7] S. Wu et. al, Sens. Actuator A, 89, (2001) 